\title{
Topological Classification of Unknotted Ring Defects
}

\author{
Hiizu Nakanishi ${ }^{1}$, Kiyoshi Hayashi ${ }^{2}{ }^{\star}$, and Hiroyuki Mori ${ }^{1}$ \\ ${ }^{1}$ Department of Physics, Faculty of Science and Technology, Keio University, \\ Yokohama 223, Japan \\ ${ }^{2}$ Department of Mathematics, Faculty of Science and Technology, Keio University, \\ Yokohama 223, Japan
}

\begin{abstract}
Unknotted ring defects in ordered media are classified in terms of the homotopy theory. It is also investigated what type of point defects will appear when a radius of the ring defect tends to zero.
\end{abstract}

\section{Introduction}

The homotopy theory of defects in ordered media has been developed in the field of condensed matter physics (see [1-4] for a review). Topologically stable defects can be classified by means of the homotopy groups of a topological space $X$ which represents internal order of a medium (order parameter space). In the theory, the configurations with defects which can be transformed into each other by continuous deformation are regarded as the same. The topological types of line defects are characterized by conjugacy classes of the first homotopy group (fundamental group), and those of point defects by automorphism classes of the second homotopy group by the action of the first homotopy group. In this paper we develop the mathematical foundation to classify defects of circular shape, or unknotted ring defects which are not penetrated by line defects (Fig. 1). We call the defects of this type the ring defects for short in this paper.

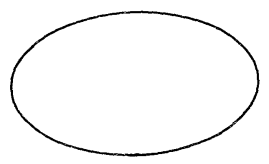

Fig. 1. An unknotted ring defect

In physical situations, ring defects appear when the two line defects which are characterized by mutually inverse elements of the first homotopy group merge. If all the parts of the two line defects approach each other evenly, they disappear at the

\footnotetext{
* Present address: Faculty of Commerce, Meiji University, Eifuku, 1-9-1, Suginami-ku, Tokyo
} 168, Japan 


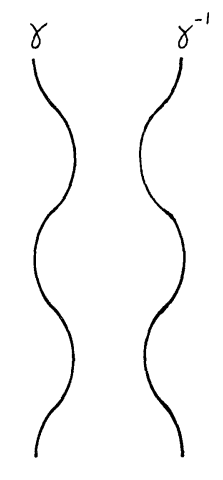

(a)

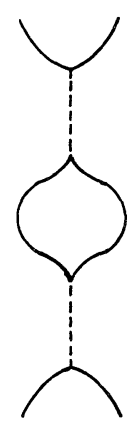

(b)

Fig. 2. a Two line defects characterized by $\gamma$ and $\gamma^{-1} \in \pi_{1}$ approach unevenly. b Some of their parts combine to disappear (the dotted lines) and a ring defect is left

same time when they merge, but if they approach unevenly and it happens that some of their parts touch each other and disappear while the others have not, ring defects are left (Fig. 2).

Although the local structure of the ring defect is nothing but that of a line defect, a ring defect is a defect localized in finite volume and does not extend to infinity like a line defect: in that sense the ring defects can be categorized as a type of point defects. In fact, some of ring defects are found to become point defects when their radii tend to zero and, as has been pointed out by Mineev [3], the characterization of the ring defects requires two parameters; one for the line defect and the other for the point defects.

The homotopy approach to the classification of ring defects has been explored by Garel [5], who studied the problem by means of the homotopy sets of mappings from tori to order parameter spaces. But these sets do not have group structure and are difficult to analyze. Besides all the elements of the sets do not necessarily correspond to ring defects themselves but some of them represent rings which are penetrated by line defects.

In the present work, we investigate the topological types of order parameter configurations with ring defects by using the homotopy sets which are somewhat different from the one studied by Garel.

\section{Order Parameter Configurations with a Single Ring Defect}

The order parameter configurations with a single ring defect are represented by mappings. $R^{3}-\Sigma \rightarrow X$, where $R^{3}$ denotes the three dimensional Euclidean space and $\Sigma$ denotes an unknotted ring, e.g. $\Sigma=\left\{(x, y, z) \in R^{3} ; x^{2}+y^{2}=1, z=0\right\}$. We assume that the order parameter space $X$ is an arcwise connected topological space and mappings are always continuous. Since $R^{3}-\Sigma$ is homotopically equivalent to the topological space $W$ which is defined as the spherical surface with the two points $N$ 


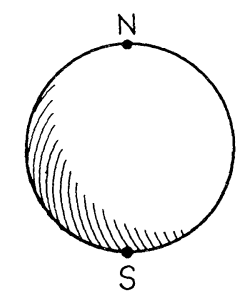

(a)

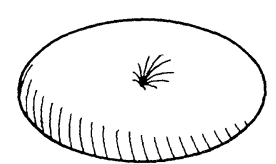

(b)

Fig. 3. a The spherical surface $S^{2}$ and b the closed surface $W$ with the north pole $N$ and south pole $S$ of $S^{2}$ shrunk into a point

and $S$ (e.g. the north and south poles) shrunk into a point

$$
W \equiv S^{2} /\{N, S\} \simeq R^{3}-\Sigma, \quad N, S \in S^{2}, \quad N \neq S
$$

(Fig. 3), the homotopy set of the mappings from $R^{3}-\Sigma$ to $X$, which is denoted by $\left[R^{3}-\Sigma ; X\right]$, is equivalent to $[W ; X]$ as a set. Therefore we can study the isolated ring defects by studying the mappings from the space $W$ to the order parameter space $X$.

In order to introduce the group structure, a base point $* \in X$ is fixed and we consider only mappings which send the base point $*_{w} \in W$ (to which $N$ and $S$ shrink) to $*$. This homotopy set $\left[W, *_{w} ; X, *\right]$ of the mappings, for which we use the notation $\tau_{2}(X, *)$, can be also defined as follows. Let $F_{\tau 2}(X, *)$ be the totality of mappings $f: I^{2} \rightarrow X$ which satisfy the condition

$$
f(u, 0)=f(u, 1), \quad f(0, v)=f(1, v)=*,
$$

where $u, v \in I \equiv[0,1]$. Then $\tau_{2}(X, *)=\pi_{0}\left(F_{\tau 2}(X, *)\right)$. The multiplication in $\tau_{2}(X, *)$ is introduced by the multiplication in $F_{\tau 2}(X, *)$, which is defined by

$$
h=f \cdot g \Leftrightarrow h(u, v)= \begin{cases}f(2 u, v) & (0 \leqq u \leqq 1 / 2) \\ g(2 u-1, v) & (1 / 2 \leqq u \leqq 1)\end{cases}
$$

and we use the notation $f^{-1} \in F_{\tau 2}(X, *)$ to denote

$$
f^{-1}(u, v)=f(1-u, v) \text {. }
$$

The same homotopy group has been considered by some mathematicians [6-8]. Then the following theorem holds.

Theorem. The homotopy group $\tau_{2}(X, *) \equiv\left[W, *_{w} ; X, *\right]$ is isomorphic to the semidirect product of $\pi_{1}(X, *)$ and $\pi_{2}(X, *)$ :

$$
\tau_{2}(X, *) \cong \pi_{1}(X, *) \ltimes \pi_{2}(X, *),
$$

by the usual action of $\pi_{1}(X, *)$ on $\pi_{2}(X, *)$. Namely, every element of $\tau_{2}(X, *)$ can be represented uniquely by a pair of $\gamma \in \pi_{1}(X, *)$ and $n \in \pi_{2}(X, *)$ as $(\gamma, n) \in \tau_{2}(X, *)$, and the multiplication is given by

$$
\left(\gamma_{1}, n_{1}\right) \cdot\left(\gamma_{2}, n_{2}\right)=\left(\gamma_{1} \cdot \gamma_{2}, \gamma_{2}^{-1}\left(n_{1}\right)+n_{2}\right),
$$


where $\gamma_{2}^{-1}\left(n_{1}\right)$ denotes the image of $n_{1} \in \pi_{2}(X, *)$ by the automorphism introduced by $\gamma_{2}^{-1} \in \pi_{1}(X, *)$. We have used the symbol + to denote the multiplication of $\pi_{2}$ since $\pi_{2}$ is Abelian.

Proof. We introduce a family of mappings $\mathrm{F}_{\pi 2}(X, *)$ as the totality of mappings $f: I^{2} \rightarrow X$ which satisfy the condition

$$
f(u, 0)=f(u, 1)=f(0, v)=f(1, v)=*,
$$

and $F_{\pi 1}(X, *)$ as the totality of mappings $f: I \rightarrow X$ which satisfy the condition

Then

$$
f(0)=f(1)=* .
$$

$$
F_{\pi 2} \stackrel{i}{\longrightarrow} F_{\pi 2} \stackrel{p}{\longrightarrow} F_{\pi 1}
$$

forms a fibre space, where $p$ is a projection given by $p(f)(u)=f(u, 0)$. In (4), we have used the abbreviation $F_{\pi 2}$ etc. to denote $F_{\pi 2}(X, *)$ etc. This fibre space has a section $s: F_{\pi 1} \rightarrow F_{\tau 2}, p \circ s=1$, which is defined by $s(g)(u, v)=g(u)$.

Then we obtain the homotopy exact sequence for this fibre space (4).

$$
\pi_{1}\left(F_{\tau 2}, *_{2}\right) \underset{s *}{\stackrel{p_{\star}}{\rightleftarrows}} \pi_{1}\left(F_{\pi 1}, *_{1}\right) \stackrel{\Delta}{\longrightarrow} \pi_{0}\left(F_{\pi 2}\right) \stackrel{i_{\star}}{\longrightarrow} \pi_{0}\left(F_{\tau 2}\right) \underset{s_{\star}}{\stackrel{p_{\star}}{\rightleftarrows}} \pi_{0}\left(F_{\pi 1}\right)
$$

leading to the exact sequence

$$
0 \rightarrow \pi_{2}(X, *) \stackrel{i_{*}}{\longrightarrow} \tau_{2}(X, *) \underset{S_{*}}{\stackrel{p_{*}}{\rightleftarrows}} \pi_{1}(X, *) \rightarrow 0
$$

with $p_{*} \circ s_{*}=1$, where the mappings $*_{1}$ and $*_{2}$ are constant mappings; $*_{1}: I \rightarrow *$ and $*_{2}: I^{2} \rightarrow *$. The sequence (5) gives (2).

We put

$$
(\gamma, n) \equiv s_{*}(\gamma) \cdot i_{*}(n)
$$

for $\gamma \in \pi_{1}(X, *)$ and $n \in \pi_{2}(X, *)$. Note that $I_{s *(\gamma)}(n)=i_{*}(\gamma(n))$, where $I_{a}(b)$ denotes an inner automorphism by $a ; I_{a}(b) \equiv a \cdot b \cdot a^{-1}$, then the multiplication rule is

$$
\begin{aligned}
\left(\gamma_{1}, n_{1}\right) \cdot\left(\gamma_{2}, n_{2}\right) & =s_{*}\left(\gamma_{1} \cdot \gamma_{2}\right) \cdot I_{s_{*}\left(\gamma_{2}^{-1}\right)}\left(i_{*}\left(n_{1}\right)\right) \cdot i_{*}\left(n_{2}\right) \\
& =s_{*}\left(\gamma_{1} \cdot \gamma_{2}\right) \cdot i_{*}\left(\gamma_{2}^{-1}\left(n_{1}\right)+n_{2}\right)=\left(\gamma_{1} \cdot \gamma_{2}, \gamma_{2}^{-1}\left(n_{1}\right)+n_{2}\right),
\end{aligned}
$$

giving (3).

A topological type of configurations with a single ring defect corresponds with an element of the homotopy set $[W ; X]$ without a base point. The correspondence between the elements of $[W ; X]$ and $\tau_{2}(X, *)=\left[W, *_{w} ; X, *\right]$ is given by the following proposition.

Proposition 1. The elements of $[W ; X]$ have natural one-to-one correspondence with the automorphism classes of $\tau_{2}(X, *)$ by an action of $\pi_{1}(X, *)$ defined below.

The action of $\gamma^{\prime} \in \pi_{1}(X, *)$ on the element $(\gamma, n) \in \tau_{2}(X, *)$ is denoted as $\gamma^{\prime}((\gamma, n))$ and given by

$$
\gamma^{\prime}((\gamma, n)) \equiv\left(\gamma^{\prime}, 0\right) \cdot(\gamma, n) \cdot\left(\gamma^{\prime}, 0\right)^{-1}
$$

where $\gamma \in \pi_{1}(X, *)$ and $n \in \pi_{2}(X, *)$. 
Proof. Let $F_{\tau 2}^{\prime}(X)$ be the totality of mappings $f: I^{2} \rightarrow X$ which satisfy

$$
f(0,0)=f(0, v)=f\left(1, v^{\prime}\right), \quad f(u, 0)=f(u, 1), \quad u, v, v^{\prime} \in I .
$$

Then $\pi_{0}\left(F_{\tau 2}^{\prime}\right)=[W ; X]$ and the inclusion $k: F_{\tau 2}(X, *) \rightarrow F_{\tau 2}^{\prime}(X)$ induce the mapping $k_{*}: \tau_{2}=\pi_{0}\left(F_{\tau 2}\right) \rightarrow[W ; X]=\pi_{0}\left(F_{\tau 2}^{\prime}\right)$.

Then we can prove that $k_{*}$ is surjective and $k_{*}^{-1}([g])$ coincides with one of the automorphism classes of $\tau_{2}(X, *)$ by the action defined above for any $[g] \in \pi_{2}\left(F_{\tau 2}^{\prime}\right)$, giving the proposition.

From Proposition 1, it is seen that two configurations with a ring defect can be transformed into each other if and only if they are characterized by the same automorphism class of $\tau_{2}(X, *)$ by the action of $\pi_{1}(X, *)$ given by (7).

Point defects are also represented by elements $(e, n)$ of $\tau_{2}$, which form a normal subgroup isomorphic to $\pi_{2}(X, *)$. This is expected because the closed surface $W$ can also enclose point defects. The fact that the ring defects are classified by the automorphism classes of $\tau_{2}$ by $\pi_{1}$ is consistent with the fact that the point defects are classified by the automorphism classes of $\pi_{2}$ by $\pi_{1}$. It is worth noting, however, that a topological type of ring defects is not ncessarily represented by a set of a conjugacy class of $\pi_{1}$ and an automorphism class of $\pi_{2}$.

\section{Correspondence Between a Ring Defect and a Point Defect}

As we have mentioned in the introduction, the ring defects are the localized defects and can be categorized in terms of the second homotopy group by considering the order parameter configuration on the sphere which encloses the defects. When the radius of the ring defect goes to zero, the singular region shrinks into a point and a point defect is left. This point defect is also characterized by the same element of $\pi_{2}(X, *)$ with the element wich characterizes the original ring defect because the deformation during the process is continuous on the sphere which encloses the defect.

We now define the mapping $\Omega: \tau_{2}(X, *) \rightarrow \pi_{2}(X, *)$ which represents the above process as follows. Let $F_{\eta 2}(X, *)$ be the totality of mappings $f: I^{2} \rightarrow X$ which satisfy

$$
f(u, 0)=f(u, 1), \quad f(0, v)=*, \quad f(1, v)=f(1,0) .
$$

Note that $F_{\eta 2} \supset F_{\tau 2} \supset F_{\pi 2}$, and there exists a homeomorphism

(Fig. 4).

$$
\omega: F_{\eta 2}(X, *) \rightarrow F_{\pi 2}(X, *)
$$

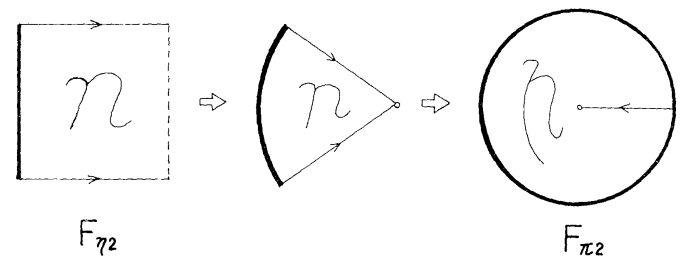

Fig. 4. Diagrams which illustrate the homeomorphism $\omega$. The heavy lines denote the regions of $I^{2}$ which are mapped into the base point $*$ of $X$ and the dashed line and the open circles are sent to some point of $X$ 
Now let $\Omega$ be the mapping $\tau_{2}(X, *) \rightarrow \pi_{2}(X, *)$ induced from the composition of the inclusion $F_{\tau 2} \subset F_{\eta 2}$ and the homeomorphism $\omega: F_{\eta 2} \approx F_{\pi 2}$. Then we have

\section{Proposition 2.}

$$
\Omega((\gamma, n))=\gamma(n) \quad \text { for } \quad(\gamma, n) \in \tau_{2}(X, *) .
$$

Proof. From $(6), \Omega((\gamma, n))=\Omega\left(s_{*}(\gamma) \cdot i_{*}(n)\right)$, and it is easily seen to be $\gamma(n)$ (Fig. 5).

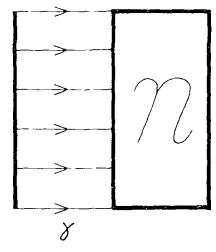

(a)

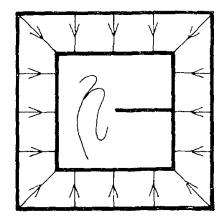

(b)

Fig. 5. The diagrams which illustrate $\Omega((\gamma, n))=\gamma(n)$. a and b represent $(\gamma, n)$ and $\Omega((\gamma, n))$, respectively

Equation (8) means that when the ring defect represented by $(\gamma, n) \in \tau_{2}$ shrinks, it becomes the point defect which is represented by $n \in \pi_{2}$ since the point defects are classified by the automorphism classes of $\pi_{2}$ by $\pi_{1}$.

\section{Ring Defects in the Presence of Other Ring or Point Defects}

The ring defects have the feature which either the line or point defects do not have, i.e. other ring or point defects can go through the ring defect. This transformation is continuous but cannot be treated as a continuous path in the space $F_{\tau 2}^{\prime}(X)$ because this continuous transformation can be considered only on the surface of the torus which encloses the ring defect but not on the space $W$.

The equivalence relation $\sim$ among the elements of $F_{\tau 2}(X, *)$ which includes the above process is defined as follows. For the elements $f$ and $f^{\prime}$ of $F_{\tau 2}(X, *), f \sim f^{\prime}$ means that they can be connected by a homotopy $h_{t}\left(t \in I, h_{0}=f, h_{1}=f^{\prime}\right)$ which satisfy

$$
h_{t}(0, v)=h_{t}(1, v), \quad h_{t}(u, 0)=h_{t}(u, 1)
$$

with $u, v \in I$. Note that $f \sim f^{\prime}$ if $f$ and $f^{\prime}$ belong to the same homotopy class of $F_{\tau 2}$, i.e. $[f]=\left[f^{\prime}\right]$, but the reverse is not necessarily true.

Proposition 3. The quotient space of $F_{\tau 2}(X, *)$ under the above equivalence relation $F_{\tau 2}(X, *) / \sim$ is isomorphic to the set of conjugacy classes of $\tau_{2}(X, *)$ as a set.

Proof. If $[f]$ and $\left[f^{\prime}\right]$ of $\tau_{2}$ where $f, f^{\prime} \in F_{\tau 2}$ belong to the same conjugacy class, there exists $g \in F_{\tau 2}$ such that $\left[f^{\prime}\right]=\left[g \cdot f \cdot g^{-1}\right]$. The homotopy $h_{t}$ can be defined by

$$
h_{t}(u, v)= \begin{cases}g(1-t+3 u, v) & (0 \leqq u \leqq t / 3) \\ f((3 u-t) /(3-2 t), v) & (t / 3 \leqq u \leqq 1-t / 3) \\ g(4-3 u-t, v) & (1-t / 3 \leqq u \leqq 1) .\end{cases}
$$

which satisfies (9), $h_{0}=f$ and $\left[h_{1}\right]=\left[f^{\prime}\right]$, therefore $f \sim h_{1} \sim f^{\prime}$. 
On the other hand if $f \sim f^{\prime}$, then there exists the homotopy $h_{t}$ with $h_{0}=f, h_{1}=f^{\prime}$, and (9). The element $g$ of $F_{\tau 2}$ which satisfies $\left[g \cdot f^{\prime} \cdot g^{-1}\right]=[f]$ can be defined as $g(u, v)=h_{u}(0, v)$.

Proposition 3 shows that two types of ring defect can be transformed into each other by continuous deformation allowing another ring or point defect to go through the ring if and only if they are characterized by the same conjugacy class of $\tau_{2}(X, *)$.

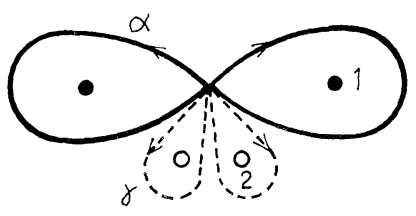

(a)

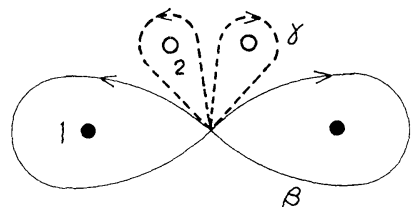

(C)

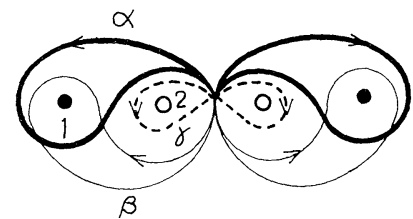

(b)

Fig. 6. The ring defect 2 whose cross section is denoted by the pair of open circles goes through the ring defect 1 denoted by the pair of filled circles; $\mathbf{a} \rightarrow \mathbf{b} \rightarrow \mathbf{c}$. The heavy and light lines (the dotted line) denote the cross section of the closed surfaces $W$, which enclose the ring defect 1 (the ring defect 2). $\alpha, \beta$, and $\gamma$ denote the elements of $\tau_{2}$ which represent the order parameter configurations on the surfaces denoted by the heavy, light, and dotted lines, respectively. The arrows indicate the direction of $u$-axis

In Fig. 6, the two pairs of filled and open circles denote cross sections of the ring defects denoted by 1 and 2 respectively, and the heavy and light lines (the dotted lines) denote the cross sections of closed surfaces $W$ which enclose the ring defect 1 (the ring defect 2). The arrows indicate the positive direction of $u$-axis. $\alpha, \beta$, and $\gamma$ denote the elements of $\tau_{2}$ which represent the configurations on the closed surfaces indicated by heavy, light, and dotted lines, respectively. From Fig. 6b, it is obvious that

$$
\alpha=\gamma \cdot \beta \cdot \gamma^{-1}
$$

and it can be seen that there are two elements $\alpha$ and $\beta$, which characterize the ring defect 1 . They may belong to different automorphism classes and $\Omega(\alpha)$ may not equal to $\Omega(\beta)$. If the ring 1 moves upwards and shrinks (Fig. 6a), then it will become the point defect characterized by $\Omega(\alpha) \in \pi_{2}$. On the other hand, if the ring 1 moves downwards and shrinks (Fig. 6c), then it will become the point defect characterized by $\Omega(\beta)$. In this sense, it might be roughly said that the ring defect 1 , which is originally characterized by $\alpha$ is transformed into the ring defect characterized by $\beta$ by means of being penetrated by the ring defect $\gamma$ (Fig. 6a-c). 


\section{Physical Examples}

The list of systems of physical interest, their order parameter spaces, and the homotopy groups $\pi_{1}, \pi_{2}$, and $\tau_{2}$ is given in Table 1 . The homotopy group $\tau_{2}$ is isomorphic to $\pi_{1}\left(\pi_{2}\right)$ if $\pi_{2}=0\left(\pi_{1}=0\right)$ since $\tau_{2}$ is a semidirect product of $\pi_{1}$ and $\pi_{2}$. Noting $\tau_{2}\left(X \times X,\left(*_{X}, *_{Y}\right)\right)=\tau_{2}\left(X, *_{X}\right) \times \tau_{2}\left(Y, *_{Y}\right)$, there are only two systems with non-trivial $\tau_{2}$ in the table; the nematic phase of liquid crystal and the dipole free $A$ phase of superfluid ${ }^{3} \mathrm{He}$. We give $\tau_{2}$ for these systems as examples. We use the same notation for groups with that of [1].

Table 1. The homotopy groups $\pi_{1}, \pi_{2}$, and $\tau_{2}$ of the topological spaces for the systems with physical interest. $Q$ denotes the quaternion group of eight elements, $\left\{ \pm 1, \pm i \sigma_{x}, \pm i \sigma_{y}, \pm i \sigma_{z}\right\}$, where $\sigma_{x}, \sigma_{y}$, and $\sigma_{z}$ are the Pauli matrices. $D_{2}$ denotes the four-element dihedral group $\subset S O(3)$, $\left\{1, R_{x}, R_{y}, R_{z}\right\}$, where $R_{x}, R_{y}$, and $R_{z}$ are the $180^{\circ}$ rotations about $x, y$, and $z$ axes, respectively

\begin{tabular}{lllll}
\hline Physical systems & Topological space & $\pi_{2}$ & $\tau_{2}$ & $\pi_{1}$ \\
\hline Planer spin & $S^{1}$ & 0 & $Z$ & $Z$ \\
Heisenberg spin & $S^{2}$ & $Z$ & $Z$ & 0 \\
Nematics & $R P^{2}$ & $Z$ & $Z_{2} \times Z$ & $Z_{2}$ \\
Biaxial nematics & $S O(3) / D_{2}$ & 0 & $Q$ & $Q$ \\
Dipole free $A$-phase $\left({ }^{3} \mathrm{He}\right)$ & $\left(S O(3) \times S^{2}\right) / Z_{2}$ & $Z$ & $Z_{4} \times Z$ & $Z_{4}$ \\
Dipole locked $A$-phase $\left({ }^{3} \mathrm{He}\right)$ & $S O(3)$ & 0 & $Z_{2}$ & $Z_{2}$ \\
Dipole free $B$-phase $\left({ }^{3} \mathrm{He}\right)$ & $S O(3) \times S^{1}$ & 0 & $Z \times Z_{2}$ & $Z \times Z_{2}$ \\
Dipole locked $B$-phase $\left({ }^{3} \mathrm{He}\right)$ & $S^{2} \times S^{1}$ & $Z$ & $Z \times Z$ & $Z$ \\
\hline
\end{tabular}

\subsection{Nematic Liquid Crystal}

The order parameter space $X$, its $\pi_{1}$, and $\pi_{2}$ are given by

$$
X=R P^{2}, \quad \pi_{1}=Z_{2}=\{0,1\}, \quad \pi_{2}=Z .
$$

Since the automorphisms of $\pi_{2}$ by $\pi_{1}$ are

$$
0(n)=n, \quad 1(n)=-n, \quad n \in \pi_{2},
$$

the multiplication law of $\tau_{2}$ is

$$
\left(\gamma_{1}, n_{1}\right) \cdot\left(\gamma_{2} \cdot n_{2}\right)= \begin{cases}\left(\gamma_{1}+\gamma_{2}(\bmod 2), n_{1}+n_{2}\right) & \text { for } \gamma_{2}=0 \\ \left(\gamma_{1}+\gamma_{2}(\bmod 2),-n_{1}+n_{2}\right) & \text { for } \gamma_{2}=1\end{cases}
$$

and inverse elements are obtained by

$$
(0, n)^{-1}=(0,-n), \quad(1, n)^{-1}=(1, n) .
$$

The expression $\gamma_{1}+\gamma_{2}(\bmod r)$ means the sum is taken in the $\bmod r$ sense. The automorphism classes by $\pi_{1}$ are

$$
A_{(m, n)}=\{(m, n),(m,-n)\}, \quad m=0,1 ; n=0,1,2, \ldots
$$


and the conjugacy classes are

$$
\begin{aligned}
& C_{(0, n)}=A_{(0, n)}, \quad n=0,1,2,3 \ldots \\
& C_{(1,0)}=\bigcup_{n=0}^{\infty} A_{(1,2 n)} \\
& C_{(1,1)}=\bigcup_{n=0}^{\infty} A_{(1,2 n+1)} .
\end{aligned}
$$

The class $A_{(0, n)}$ gives the classification of point defects.

\subsection{Dipole Free A-Phase of ${ }^{3} \mathrm{He}$}

For this system

$$
\begin{aligned}
& X=\left(S O(3) \times S^{2}\right) / Z_{2}, \\
& \pi_{1}=Z_{4}=\{0,1,2,3\}, \quad \pi_{2}=Z .
\end{aligned}
$$

and the automorphisms of $\pi_{2}$ by $\pi_{1}$ are

$$
0(n)=2(n)=n, \quad 1(n)=3(n)=-n .
$$

The multiplications and inverse elements are given by

and

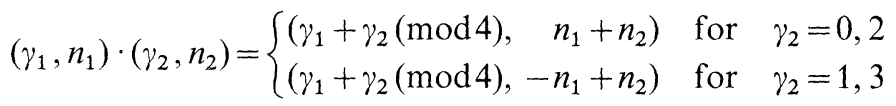

$$
\begin{array}{ll}
(0, n)^{-1}=(0,-n), & (1, n)^{-1}=(3, n), \\
(2, n)^{-1}=(2,-n), & (3, n)^{-1}=(1, n) .
\end{array}
$$

The automorphism classes by $\pi_{1}$ are

$$
\begin{aligned}
A_{(m, n)}=\{(m, n),(m,-n)\}, \quad m & =0,1,2,3 ; \\
n & =0,1,2,3, \ldots
\end{aligned}
$$

and the conjugacy clases are

$$
\begin{aligned}
& C_{(0, n)}=A_{(0, n)}, \quad n=0,1,2,3, \ldots \\
& C_{(1,0)}=\bigcup_{n=0}^{\infty} A_{(1,2 n)} \\
& C_{(1,1)}=\bigcup_{n=0}^{\infty} A_{(1,2 n+1)} \\
& C_{(2, n)}=A_{(2, n)}, \quad n=0,1,2,3, \ldots \\
& C_{(3,0)}=\bigcup_{n=0}^{\infty} A_{(3,2 n)} \\
& C_{(3,1)}=\bigcup_{n=0}^{\infty} A_{(3,2 n+1)} .
\end{aligned}
$$

The class $A_{(0, n)}$ gives the classification of point defects. 


\section{Summary and Discussions}

We have investigated the topological classification of ring defects by analyzing the homotopy group $\tau_{2}$ of the mappings from the space $W$, which is homotopically equivalent to $R^{3}-\Sigma$, to the order parameter space. It has been shown that $\tau_{2}$ is a semidirect product of $\pi_{1}$ and $\pi_{2}$, which is the manifestation of the duality of the ring defects, namely, the local structure of the singularity is identical with that of the line defects while the defect is confined in finite volume like a point defect. The topological types of isolated ring defects correspond to the automorphism classes of $\tau_{2}$ by the $\pi_{1}$-action, whereas one type of the ring defects can be transformed into another type which is characterized by the conjugate element of the original one in the presence of other ring or point defects if they are allowed to go through the ring defect.

For both physical examples shown in Sect. 5, the automorphism classes of $\tau_{2}$, (11) and (12), coincide with the direct products of the conjugacy classes of $\pi_{1}$ and the automorphism classes of $\pi_{2}$ since $\pi_{1}$ is Abelian. But it is not true in general. When $X$ is not 2-simple and has non-Abelian $\pi_{1}$, the two sets do not necessarily coincide and the number of topological type of ring defects can be greater than that of line defects times that of ring defects although we have no such interesting physical examples in Table $1^{1}$.

The multiplication of two elements of $\tau_{2}$ is easily seen to give the type of ring defect which is obtained when the two ring defects merge in the way shown in Fig. 7a. When the multiplication of two automorphism classes does not result in a single automorphism class, the result of the defect combination will depend on the path along which they merge in the presence of other line defects, as in the situation for the line defects (Fig. 7b).

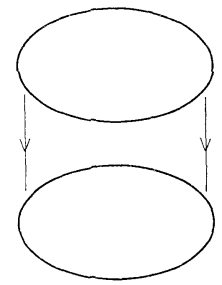

(a)

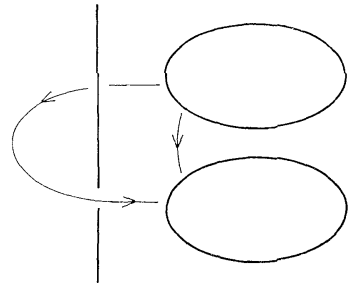

(b)

Fig. 7. a Two ring defects combine into one. b Two different paths along which two ring defects combine in the presence of a line defect

Some of the ring defects leave point defects when the shrink. The theory predicts that the elements which belong to the same conjugacy class can result in different point defects [see (8), (12), ans (14)]. However, this is not a contradiction and a similar situation occurs for the point defects in the presence of the line defects, i.e.

\footnotetext{
${ }^{1}$ We can easily construct a mathematical example for which the two sets do not coincide. For example, if we define the action by $D_{2}$ on $S O(3)$ as the left multiplication and that on $S^{2}$ as $1, R_{x}: \mathbf{r} \in S^{2} \mapsto \mathbf{r}$ and $R_{y}, R_{z}: \mathbf{r} \mapsto-\mathbf{r}$, then for $X \equiv\left(S O(3) \times S^{2}\right) / D_{2}, \pi_{1}(X)=Q$ and $\pi_{2}(X)=Z$. The $\pi_{1}$-action on $\pi_{2}$ is $\pm 1, \pm i \sigma_{x}: n \in Z \mapsto n$ and $\pm i \sigma_{y}, \pm i \sigma_{z}: n \mapsto-n$
} 
the point defects can be created or annihilated by being brought around the line defect. Analogously, in the presence of another ring defect or point defect, the ring defect results in different types of point defects depending on whether it shrinks directly or after the ring or point defects pass through the ring defect.

Some extension of the present theory is possible. A ring defect in the four dimensional space can be treated by a simple extension of the theory and can be interpreted as a pair creation and annihilation of point defects. The theory can also apply to the classification of nonsingular texture of a "ring soliton" which are embedded in the uniform configuration.

Acknowledgement. The authors are grateful to Dr. M. Murayama for helpful suggestions.

\section{References}

1. Mermin, N.D.: The topological theory of defects in ordered media. Rev. Mod. Phys. 51, 591-648 (1979)

2. Michel, L.: Symmetry defects and broken symmetry. Configurations. Hidden symmetry. Rev. Mod. Phys. 52, 617-651 (1980)

3. Mineev, V.P.: Topologically stable defects and solitons in ordered media. Sov. Sci. Rev. A2, 173-246 (1980)

4. Trebin, H.R.: The topology of non-uniform media in condensed matter physics. Adv. Phys. 31, 195-254 (1982)

5. Garel, A.T.: Boundary conditions for textures and defects. J. Phys. 39, 225-229 (1978)

6. Abe, M.: Über die stetigen Abbildungen der $n$-Sphäre in einen metrischen Raum. Jpn. J. Math. 16, 169-176 (1939)

7. Fox, R.H.: Torus homotopy groups. Proc. Natl. Acad. Sci. (USA) 31, 71-74 (1945)

8. Hu, S.-T.: On spherical mappings in a metric space. Ann. Math. 48, 717-734 (1947)

Communicated by J. Fröhlich

Received May 13, 1987; in revised form December 31, 1987 
